NISSUNA UMANA INVESTIGAZIONE SI PUO DIMANDARE VERA SCIENZIA S'ESSA NON PASSA PER LE MATEMATICHE DIMOSTRAZIONI LEONARDO DA VINCI

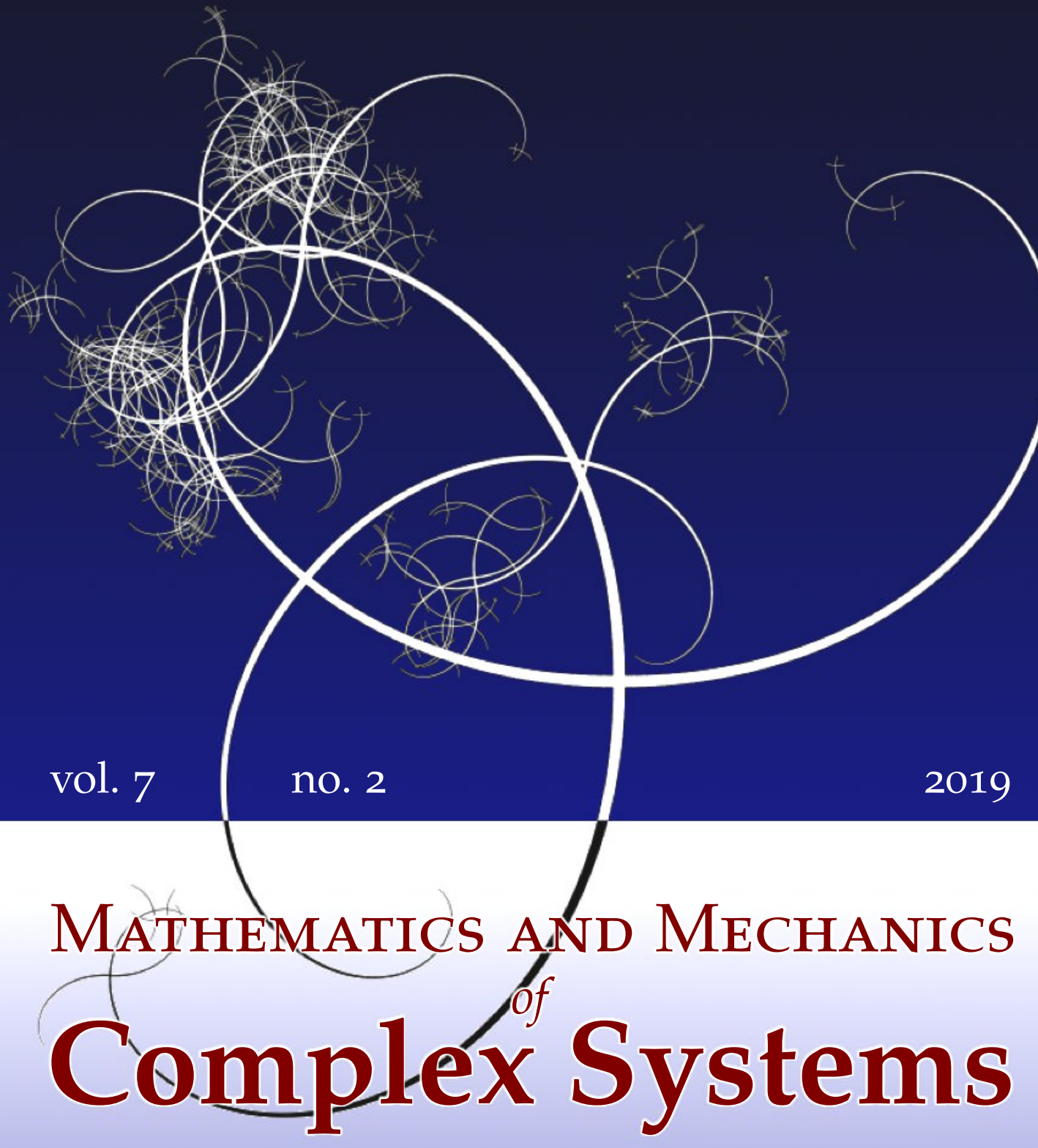

IVAN Giorgio AND Dionisio Del Vescovo

ENERGY-BASED TRAJECTORY TRACKING AND VIBRATION CONTROL

FOR MULTILINK HIGHLY FLEXIBLE MANIPULATORS 


\title{
ENERGY-BASED TRAJECTORY TRACKING AND VIBRATION CONTROL FOR MULTILINK HIGHLY FLEXIBLE MANIPULATORS
}

\author{
IVAn Giorgio AND Dionisio Del Vescovo
}

"I accept nothing on authority. A hypothesis must be backed by reason, or else it is worthless." —Isaac Asimov, "Reason", I, Robot (1950)

In this paper, a discrete model is adopted, as proposed by Hencky for elastica based on rigid bars and lumped rotational springs, to design the control of a lightweight planar manipulator with multiple highly flexible links. This model is particularly suited to deal with nonlinear equations of motion as those associated with multilink robot arms, because it does not include any simplification due to linearization, as in the assumed modes method. The aim of the control is to track a trajectory of the end effector of the robot arm, without the onset of vibrations. To this end, an energy-based method is proposed. Numerical simulations show the effectiveness of the presented approach.

\section{Introduction}

Discrete formulations for continuous systems, usually, are required to avoid the difficulty associated with solving partial differential equations and with satisfying their boundary conditions. In order to eliminate the spatial dependence from the problem and, thus, to deal with a set of ordinary differential equations which approximates the distributed-parameter system, a proper discretization can be made. The adopted techniques for this purpose can be grouped into two main categories: procedures based on the approximation of the solution by means of a finite series of given functions and approaches resulting in lumped parameter systems. In particular, to model multilink flexible arms, many authors employed the assumed modes method [Bellezza et al. 1990; Khorrami et al. 1991; De Luca and Siciliano 1991] and the finite element formulation [Ramachandran et al. 1992; Sharf 1996], both of which belong to the first group; other authors prefer the lumped-parameter approach [Rubinstein 1999; Dupac and Noroozi 2014; Giorgio and Del Vescovo 2018] for ease of use.

\section{Communicated by Francesco dell'Isola.}

MSC2010: primary 70E60; secondary 70E55, 70KXX, 74KXX, 74SXX.

Keywords: nonlinear elastica, discrete modeling, flexible robot arms, energy-based control. 
In the assumed modes method, the solution representing the transverse deflection of the flexible beams is expanded into a finite summation of space-dependent functions satisfying the geometric boundary conditions (i.e., admissible functions) with time-dependent coefficients, i.e., the generalized coordinates. In this method, the set of admissible functions is much larger than the set of eigenfunctions (i.e., normal modes); thus, the solution is affected by an error related to the choice of the admissible functions. This error increases with the eigenfrequencies, and to reduce it, a relatively large number of the terms of the truncated series is advisable to be employed. Although this method has been widely used in the literature, it is only applicable to discretize linear systems. Nevertheless, the equations related to a multilink manipulator are strongly nonlinear. To overcome this issue, a simplified assumption should be made considering that the motion could be characterized by two distinct time scales: one "slower" for the overall motion and one "faster" for the superimposed vibration. Therefore, the key idea is to linearize the nonlinear equations around any configuration reached during the motion, or in other words it is possible to assume that, in a reasonably small time interval, the overall motion is sufficiently slow and the current configuration is almost time-constant when compared to the vibration. In view of these considerations, the problem of the time dependence of the frequency equation for planar multilink flexible arms [De Luca and Siciliano 1991] could be solved by keeping constant the boundary conditions due to mass terms for a fixed arm configuration. But in this way, many admissible functions for the transverse displacement discretization are required, which increases the number of discrete Lagrange equations obtained. An alternative way, to keep the number of final ordinary equations lower, consists of updating the values of the mass and inertia coefficients which appear in the boundary conditions at each time step, thinking of them, in view of the two time scales, as constant parameters and, hence, having a suitable approximation for the admissible functions (see, e.g., [Giorgio et al. 2019]).

The finite element formulation has the same basic idea as the assumed modes method; the main difference between the two approaches lies in the nature of the admissible functions. In the assumed modes method, the trial functions are defined on the entire domain; in the finite element method, they are functions defined on compact subdomains of the system, namely, the "finite elements". This particular subdivision of the entire domain allows one to use simpler admissible functions, typically low-degree polynomials (i.e., interpolating functions). Unfortunately, the greater flexibility of this method entails more degrees of freedom and, ultimately, a greater number of discrete equations than those required by the assumed modes method. Besides, analogously to the previous method, the natural frequencies computed with the finite element analysis are overestimated. However, from a computational point of view, this method requires fewer mathematical operations; 
therefore, it is particularly suited for dynamic model-based online controller implementations [Theodore and Ghosal 1995]. Although the finite element method can be identified as a different version of the assumed modes method, it can be generalized to be used in a wider context, in particular, when nonlinear effects arise as for a multilink manipulator [Sharf 1996; Eugster et al. 2014; Luongo and D'Annibale 2013]. To address some issues related to failures in convergence that are occasionally experienced, some authors have proposed a mixed formulation, based on both stress and displacement degrees of freedom, which appears very promising in this respect [Hodges 1990; Garcea et al. 1998]. However, this reformulation of the problem involves a greater complexity of modeling. An alternative approach is based on the isogeometric formulation proposed by [Hughes et al. 2005] and further developed by many other research groups (see, e.g., [Greco and Cuomo 2013; Balobanov et al. 2016; Cazzani et al. 2016; Weeger et al. 2013] and more recently [Greco et al. 2017; Yildizdag et al. 2018]). The key concept of the isogeometric analysis consists of using B-splines or NURBS curves both for representing the geometry of the system and as interpolating functions in the finite element method. Some examples in which such a method has been adopted within the framework of nonlinear structural vibration analysis produced very promising results. In addition, this formulation allows one to use fewer elements than the classical polynomial-based finite element analysis without losing accuracy.

Lumped parameter models, applied to a beam-like structure, simplify the description of the behavior of one-dimensional continuum systems into an articulated chain consisting of a discrete number of rigid bodies that approximate the behavior of the distributed system under the assumption that all interactions between the rigid segments take place via frictionless hinges with elastic rotational spring and possibly dampers [Wang et al. 2015; Kocsis et al. 2017; Turco et al. 2016]. Therefore, the lumped-parameter approach is the only method born naturally nonlinear. The first author, to our knowledge, to propose the replacement of the continuum structure with a discrete one was Hencky in 1920, who studied the buckling of a beam. To solve dynamical problems, also the distributions of the mass should be discretized, sometimes by taking into account the inertial properties of the rigid segments [Rubinstein 1999] and other times by considering lumped masses in the fictitious joints [Feliu et al. 1992]. In order to improve performances of trajectory tracking for the end effector of a multilink manipulator, in this paper, for its simplicity and nonlinear character, the latter method is adopted. Indeed, improving performances means decreasing the overall time of the motion for the task; this makes the hypothesis of linearization characterizing the assumed modes method no longer satisfied in many applications. In any case, all the methods described have advantages and disadvantages. Therefore, the wise judgment of the researcher should be the guide in a case-by-case choice, depending on the particular application. 
The aim of the work concerns the modeling and control of flexible planar multilink arms. Many researchers have been recently paying attention to this kind of system because of its increasing importance within the applications of so-called soft robots. In addition, it is conceivable to use the present formulation to model polymeric chains as well as some highly flexible structures in the space applications. Thus, the considered model may find applications from nano- until macroscales. The key point of the paper is using flexible hinges and their description using finite rotations within the discrete modeling of the multilink arm. In particular, an energy-based method is proposed to control the trajectory of the robot arm end effector, without the onset of vibrations. The paper is organized as follows. First, Section 2 gives the kinematic description for the introduced discrete method applied to planar multilink flexible arms in a recursive form and, accordingly to a Lagrangian approach, the equations of motion. Section 3 is devoted to describing the proposed control strategy using a trajectory planning described in Section 3.1. Section 4 reports simulation results for a two-link flexible arm. Conclusions are presented in the final section.

\section{Modeling}

A planar articulated kinematic chain of $n_{\ell}$ flexible links of length $\ell_{i}$ - connected by revolute joints - is considered. The $i$-th flexible link is studied as a lumped parameter system consisting of $n_{e}$ successive rigid rods of length $\eta_{j}$ (with $j=1, \ldots, n_{e}$ ) and connected by torsional springs. For the sake of simplicity, it is assumed that the rods are arranged along a straight line in the undeformed configuration. Lumped masses are placed at the boundaries of each rigid segment. As a result, each link is a system of $n_{e}$ massless rigid rods and $n_{e}+1$ point masses. The flexural stiffness of links is given by torsional springs. Finally, actuators and payload are modeled as rigid bodies. The center of mass of each actuator is located at the joint point of the link, while the payload barycenter is located at the tip of the whole system.

In order to describe motions of the multilink arm, $n_{\ell}$ moving reference frames are introduced, i.e., one for each flexible link in the spirit of the Denavit-Hartenberg convention. The $x$-axis (abscissa) of the $i$-th link is oriented as its first rigid segment and its origin, whose position vector expressed in the global frame is denoted by $\boldsymbol{r}_{i}$, coincides with the position of the actuated joint. Therefore, such moving reference frames can be referred to as "pseudoclamped" frames (see Figure 1).

The following two Lagrangian coordinates are introduced for the multilink arm. First is the joint angle, $\vartheta_{i}(t)$, i.e., the relative rotation between the first segment of the $i$-th link and the last segment of the preceding link, which meet in the $i$-th joint. The angle $\vartheta_{1}(t)$ is evaluated with respect to the $X_{0}$-axis of the global reference 


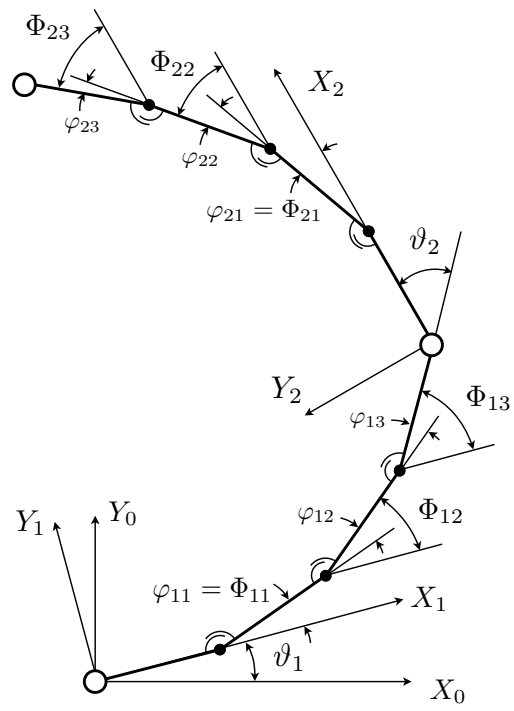

Figure 1. Discrete system corresponding to a planar two-link flexible arm.

frame. Second is $\Phi_{i j}(t)$, with $i=1, \ldots, n_{\ell}$ and $j=1, \ldots, n_{e}$, the relative angle of the $j$-th segment with respect to the $x$-axis of $i$-th reference frame.

The relative angles between adjacent segments are defined as $\varphi_{i j}(t)=\Phi_{i j}(t)-$ $\Phi_{i j-1}(t)$, with $\varphi_{i 1}(t)=\Phi_{i 1}(t)$. Within the local reference frame related to link $i$, the position vector of any end point of rigid segments is denoted as ${ }^{i} \boldsymbol{p}_{i j}(t)$. Therefore, within the same $i$-th frame, the relation ${ }^{i} \boldsymbol{r}_{i+1}(t)={ }^{i} \boldsymbol{p}_{i n_{e}}(t)$ is valid owing to the hinge constraint requiring the origin of the $(i+1)$-th frame and the end point of the $i$-th link to have the same position in space. By introducing the rotation matrices

$$
R_{i}=\left[\begin{array}{rr}
\cos \vartheta_{i} & -\sin \vartheta_{i} \\
\sin \vartheta_{i} & \cos \vartheta_{i}
\end{array}\right], \quad \widehat{R}_{i j}=\left[\begin{array}{rr}
\cos \Phi_{i j} & -\sin \Phi_{i j} \\
\sin \Phi_{i j} & \cos \Phi_{i j}
\end{array}\right]
$$

the position of the generic point of the kinematic chain can be written in the local reference frame using the recursive formula

$$
{ }^{i} \boldsymbol{p}_{i j}={ }^{i} \boldsymbol{p}_{i j-1}+\widehat{R}_{i j-1}\left(\Phi_{i j-1}\right)^{i} \boldsymbol{p}_{i 1}, \quad{ }^{i} \boldsymbol{p}_{i 1} \equiv\left[\eta_{i}, 0\right]^{T},
$$

and in the global reference frame as

$$
\boldsymbol{p}_{i j}=\boldsymbol{r}_{i}+Q_{i}{ }^{i} \boldsymbol{p}_{i j}
$$


where the global transformation operator $Q_{i}$ (with $i=\left\{1, \ldots, n_{\ell}\right\}$ ) is obtained by the recursive equation

$$
Q_{i}=\widehat{Q}_{i-1} R_{i}\left(\vartheta_{i}\right), \quad\left\{\begin{array}{l}
\widehat{Q}_{0}=I, \\
\widehat{Q}_{i}=Q_{i} \widehat{R}_{i n_{e}}\left(\Phi_{i n_{e}}\right),
\end{array}\right.
$$

$I$ being the identity operator. Similarly, the following expression is fulfilled by the origins of the local reference frames:

$$
\boldsymbol{r}_{i+1}=\boldsymbol{r}_{i}+Q_{i}{ }^{i} \boldsymbol{r}_{i+1}
$$

The angular velocity of the $i$-th reference frame reads as

$$
\dot{\alpha}_{i}(t)=\sum_{j=1}^{i} \dot{\vartheta}_{j}(t)+\sum_{k=1}^{i-1} \dot{\Phi}_{k n_{e}}(t)
$$

while the angular velocity of the payload can be evaluated as $\dot{\alpha}_{p}(t)=\dot{\alpha}_{n_{\ell}}(t)+\dot{\Phi}_{n_{\ell} n_{e}}$ and, finally, the velocity vector of any point of the kinematic chain is easy to compute as

$$
\dot{\boldsymbol{p}}_{i}=\dot{\boldsymbol{r}}_{i}+\dot{Q}_{i}{ }^{i} \boldsymbol{p}_{i}+Q_{i}{ }^{i} \dot{\boldsymbol{p}}_{i}
$$

The total kinetic energy of the mechanical system stemming from the above assumptions is

$$
\mathfrak{K}=\sum_{i=1}^{n_{\ell}}\left(\mathfrak{K}_{h i}+\mathfrak{K}_{\ell i}\right)+\mathfrak{K}_{p}
$$

where the following terms can be recognized:

(1) the kinetic energy of the actuator located at the $i$-th joint and characterized by mass $m_{h i}$ and moment of inertia $J_{h i}$,

$$
\mathfrak{K}_{h i}=\frac{1}{2} m_{h i} \dot{\boldsymbol{r}}_{i}^{T} \dot{\boldsymbol{r}}_{i}+\frac{1}{2} J_{h i} \dot{\alpha}_{i}^{2},
$$

(2) the kinetic energy of each link with lumped mass $m_{i j}$,

$$
\mathfrak{K}_{\ell i}=\frac{1}{2} \sum_{j=1}^{n_{e}} m_{i j} \dot{\boldsymbol{p}}_{i j}^{T} \dot{\boldsymbol{p}}_{i j},
$$

(3) and the kinetic energy of the payload with mass $m_{p}$ and moment of inertia $J_{p}$,

$$
\mathfrak{K}_{p}=\frac{1}{2} m_{p} \dot{\boldsymbol{p}}_{n_{\ell} n_{e}}^{T} \dot{\boldsymbol{p}}_{n_{\ell} n_{e}}+\frac{1}{2} J_{p} \dot{\alpha}_{p}^{2} .
$$

The elastic potential energy is assumed to be

$$
\mathfrak{U}=\sum_{i=1}^{n_{\ell}} \sum_{j=1}^{n_{e}} b_{i}\left[1-\cos \varphi_{i j}\right]
$$


where $b_{i}=Y_{i} j_{i} / \eta_{i}$ is the lumped bending stiffness of the introduced torsional springs, $Y_{i}$ is the Young modulus of constituting material, and $j_{i}$ is the second moment of area of the link's cross-section. We remark that this model is able to take into account also nonlinear elastic behaviors of the system; indeed, it has been shown [dell' Isola et al. 2016] that such a model, in the homogenized limit, converges to that of a nonlinear beam [Turco 2018; Pietraszkiewicz and Eremeyev 2009; Spagnuolo and Andreaus 2019] being shear undeformable, suitable for the description of problems involving large displacements and large deformations (see [Rosi et al. 2018; Placidi et al. 2017; Baroudi et al. 2019] for more details on methods for obtaining material parameters), and whose deformation energy density depends only upon the exact curvature. Note that the linearized form of (9) is simply a quadratic form in the relative angle $\varphi_{i j}$. Here, the general expression (9) of the strain energy is considered, because we are dealing with situations in which the kinetic energy contains nonnegligible nonquadratic terms. It would not be coherent to consider a quadratic approximation only for the elastic energy (while not for the kinetic energy) and, in any case, it would not lead to any significant simplification. Besides, the linearization of corresponding equation is a mathematical trick which aims to obtain solutions more easily, but it is not always possible.

It is worth noting that the choice of $\Phi_{i j}$ variables implies a simpler expression for the kinetic energy, while the choice of $\varphi_{i j}$ variables implies a simpler expression for the elastic energy; therefore, since the kinetic energy represents the more complex term in the Lagrangian, the first set of variables is used.

The equations of motion obeyed by the considered planar $n_{\ell}$-link flexible arm can be derived by introducing the Lagrangian:

$$
\mathfrak{L}=\mathfrak{K}-\mathfrak{U} .
$$

Possibly, some viscous dissipation can also be introduced by means of a Rayleigh potential as

$$
\mathfrak{D}=\sum_{i=1}^{n_{\ell}} \sum_{j=1}^{n_{e}} \frac{1}{2} c_{i} \dot{\varphi}_{i j}^{2}
$$

By differentiating $\mathfrak{D}$ with respect to the velocities $\dot{\varphi}_{i j}$ and multiplying the result by the virtual angular displacements $\delta \varphi_{i j}$, the work done by dissipative moments $\sum_{i=1}^{n_{\ell}} \sum_{j=1}^{n_{e}} c_{i} \dot{\varphi}_{i j} \delta \varphi_{i j}$ can be evaluated. This work becomes

$$
\sum_{i=1}^{n_{\ell}} \sum_{j=1}^{n_{e}} c_{i}\left(\dot{\Phi}_{i j}-\dot{\Phi}_{i j-1}\right) \delta\left(\Phi_{i j}-\Phi_{i j-1}\right)
$$

when it is expressed in terms of the chosen Lagrangian coordinates and, hence, the dissipative terms to be added to the equations of motion are easily obtained. 
In order to model friction which may occur within the joints, a Lund-Grenoble model is employed. The reason for such a choice lies in the fact that it is able to take into account the predominant nonlinear effects involved in such phenomena like presliding displacement, stick-slip motion, the Stribeck effect, and so forth [Canudas de Wit et al. 1995]. Since this formulation is based on a dynamic model, the friction torque evolves according to a differential equation conceived to match experimental measures. A simple evolution rule for the friction torques $\tau_{f i}$ can be assumed as

$$
\frac{\mathrm{d} \tau_{\mathfrak{f} i}}{\mathrm{~d} t}=k_{i} \dot{\vartheta}_{i}\left(1-\frac{\tau_{f i}}{\tau_{L}\left(\dot{\vartheta}_{i}\right)} \operatorname{sign} \dot{\vartheta}_{i}\right)
$$

where $\tau_{L}\left(\dot{\vartheta}_{i}\right)=\tau_{C}+\left(\tau_{S}-\tau_{C}\right) \exp \left[-\left(\dot{\vartheta}_{i} / \nu_{S}\right)^{2}\right]$ is the limit torque related to the Stribeck effect. The quantity $\tau_{S}$ is the static friction torque, $\tau_{C}$ is the Coulomb friction torque, and $v_{s}$ represents the Stribeck velocity.

\section{Control strategy}

In this section, an energy-based control approach is proposed to solve a trajectorytracking and vibration control problem. In particular, given a family of desired trajectories for the tip of each link, $\boldsymbol{x}_{\mathrm{des} i}(t)$, we propose to implement a strategy of control based on the potential energy

$$
U\left(\vartheta_{i}, \varphi_{i j}\right)=\sum_{i=1}^{n_{\ell}} \frac{1}{2} K_{c i}\left\|\boldsymbol{p}_{i n_{e}}\left(\vartheta_{i}, \varphi_{i j}\right)-\boldsymbol{x}_{\mathrm{des} i}(t)\right\|^{2}
$$

where $K_{c i}$ are positive constant control parameters. The virtual work related to that "control action" can be easily expressed as

$$
\delta U=\sum_{i=1}^{n_{\ell}} \frac{\partial U}{\partial \vartheta_{i}} \delta \vartheta_{i}+\sum_{i=1}^{n_{\ell}} \sum_{j=1}^{n_{e}} \frac{\partial U}{\partial \varphi_{i j}} \delta \varphi_{i j}
$$

where the negative gradient of the potential $U$, whose components are $-\partial U / \partial \vartheta_{i}$ and $-\partial U / \partial \varphi_{i j}$, is the generalized conservative action which does work on the Lagrangian coordinates $\vartheta_{i}$ and $\varphi_{i j}$. Indeed, these actions can be interpreted as joint torques and lumped moments which bend the link in correspondence of the connections between adjacent rigid segments. Therefore, measuring the variables $\vartheta_{i}$ and $\varphi_{i j}$, it is possible to compute these generalized actions and to feed them back to the multilink in order to mimic the above mentioned potential $U$. Regarding the design phase of the control, both the measurements of variables $\varphi_{i j}$ and the feeding of applied moments $\partial U / \partial \varphi_{i j}$ can be implemented by means of piezoelectric patches located on the ends of the segments in which the system has been discretized. We briefly recall, indeed, that piezoelectric transducers are simultaneously able to be employed as both sensors and actuators [Alessandroni et al. 
2005; Lumentut and Howard 2015; Chróścielewski et al. 2019; Lossouarn et al. 2015] and they can nowadays exert forces up to $70 \mathrm{kN}$ [Aminzahed et al. 2017]. Thus, they are particularly suited for this kind of control, which results in being colocated in the sense of [Cannon 1984]. In addition, the present technique can be classified in the framework of a virtual passive approach. As it is characterized by a passive constitutive law, it exhibits with respect to a purely active approach the main advantage of being unconditionally stable [Juang and Phan 1992].

To improve the performance of the control, we can also introduce a dissipative control action by means of the Rayleigh function

$$
D\left(\vartheta_{i}, \varphi_{i j}, \dot{\vartheta}_{i}, \dot{\varphi}_{i j}\right)=\sum_{i=1}^{n_{\ell}} \frac{1}{2} C_{c i}\left\|\dot{\boldsymbol{p}}_{i n_{e}}-\dot{\boldsymbol{x}}_{\mathrm{des} i}(t)\right\|^{2}
$$

expressed in terms of the relative velocities between the moving ends of each link and the desired points of the trajectory. The quantities $C_{c i}$ are positive constant control parameters related to the introduced damping. Analogously to what has been done above, the virtual work done by the dissipative control action is

$$
\delta D=\sum_{i=1}^{n_{\ell}} \frac{\partial D}{\partial \dot{\vartheta}_{i}} \delta \vartheta_{i}+\sum_{i=1}^{n_{\ell}} \sum_{j=1}^{n_{e}} \frac{\partial D}{\partial \dot{\varphi}_{i j}} \delta \varphi_{i j},
$$

the quantities $-\partial D / \partial \dot{\vartheta}_{i}$ and $-\partial D / \partial \dot{\varphi}_{i j}$ being the new generalized actions to be added to the previous ones. In order to implement this additional contribution, however, it is necessary to employ further velocity sensors for $\dot{\vartheta}_{i}$ and $\dot{\varphi}_{i j}$.

3.1. Trajectory planning. In this section, in order to analyze the capabilities of the proposed control law, a two-link arm is considered and two geometric paths to be followed by the end effector $\boldsymbol{x}_{\operatorname{des} n_{\ell}}=\boldsymbol{x}_{\operatorname{des} n_{\ell}}(u)$ are introduced, defined by means of a parametrization in terms of the scalar $u$ : a rectilinear and a closed loop path. Each geometric path is tracked according to the motion law $u=u(t)$. In particular, a polynomial function, whose coefficients have been determined in order to satisfy proper boundary conditions, i.e., null values up to the time derivative of the jerk, is employed as

$$
u_{\mathrm{des}}(t)=u_{0}+A_{\mathrm{des}}\left[126\left(t / T_{s}\right)^{5}-420\left(t / T_{s}\right)^{6}+540\left(t / T_{s}\right)^{7}-315\left(t / T_{s}\right)^{8}+70\left(t / T_{s}\right)^{9}\right]
$$

where $u_{0}$ is the value at the initial instant, $A_{\text {des }}$ is the amplitude, and $T_{s}$ is the time of the task.

Once the trajectory is assigned to the end effector, the trajectories for the intermediate joint points are obtained by assuming a rigid motion for the corresponding links. 


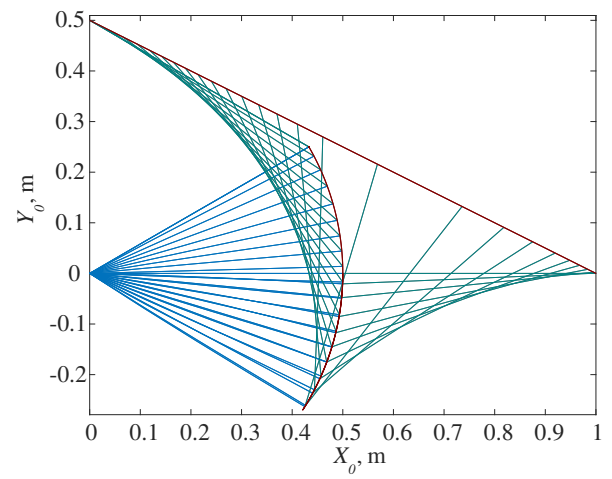

Figure 2. Straight trajectory.

Rectilinear trajectory. The considered rectilinear trajectory is defined as

$$
\left\{\begin{array}{l}
x_{\mathrm{des} 2}=\ell_{1}+\ell_{2}-u_{\mathrm{des}} \cos \alpha \\
y_{\mathrm{des} 2}=u_{\mathrm{des}} \sin \alpha
\end{array}\right.
$$

where $u_{0}=0$ and $A_{\mathrm{des}}=\sqrt{\ell_{1}^{2}+\left(\ell_{1}+\ell_{2}\right)^{2}}$. The parameter $u_{\mathrm{des}}$ ranges from 0 to $A_{\text {des }}$ (achieved at time $T_{s}$ ); this means that initially the manipulator is arranged along the axis $X_{0}$, and finally the end effector reaches the $Y_{0}$ axis at the point $\left(0, \ell_{1}\right)$. Hence, $\alpha=\operatorname{atan}\left[\ell_{1} /\left(\ell_{1}+\ell_{2}\right)\right]$.

Closed loop trajectory. The closed loop trajectory is defined as

$$
\left\{\begin{array}{l}
x_{\mathrm{des} 2}=\frac{1}{8}\left[\cos u_{\mathrm{des}}+\sqrt{3+2 \sin u_{\mathrm{des}}-\sin \left(u_{\mathrm{des}}\right)^{2}}\right], \\
y_{\mathrm{des} 2}=\frac{1}{4}\left(1+\sin u_{\mathrm{des}}\right)+\ell_{1} / 5
\end{array}\right.
$$

where $u_{0}=3 / 2 \pi$ and $A_{\text {des }}=2 \pi$. The parameter $u_{\text {des }}$ ranges from $u_{0}$ to $u_{0}+A_{\text {des }}$. These values are chosen so as to have the end effector initially on the $Y_{0}$ axis - at the point $\left(0, \ell_{1} / 5\right)$ - and returning to the same position at the end of the motion.

\section{Numerical simulations}

In performing numerical simulations we have considered the links in the two-link planar manipulator having length $\ell_{1}=\ell_{2}=0.5 \mathrm{~m}$ and having a rectangular crosssection of size $2 \times 50 \mathrm{~mm}$; the discretization of each link is made by dividing it into four segments. The Young modulus of the material constituting the link is $Y_{b}=200 \mathrm{GPa}$, and thus, the bending stiffnesses are $b_{1}=b_{2}=53.3 \mathrm{~N} \mathrm{~m}$; the lumped masses are assumed to be $m_{i j}=0.0981 \mathrm{~kg}$ for the inner points and half of this value for the outermost points; the payload mass and moment of inertia are $m_{p}=0.1 \mathrm{~kg}$ and $J_{p}=0.005 \mathrm{~kg} \mathrm{~m}^{2}$, respectively; the hub mass and moment of inertia are $m_{h 1}=$ $m_{h 2}=1 \mathrm{~kg}$ and $J_{h 1}=J_{h 2}=0.1 \mathrm{~kg} \mathrm{~m}^{2}$, respectively. The considered dissipation 

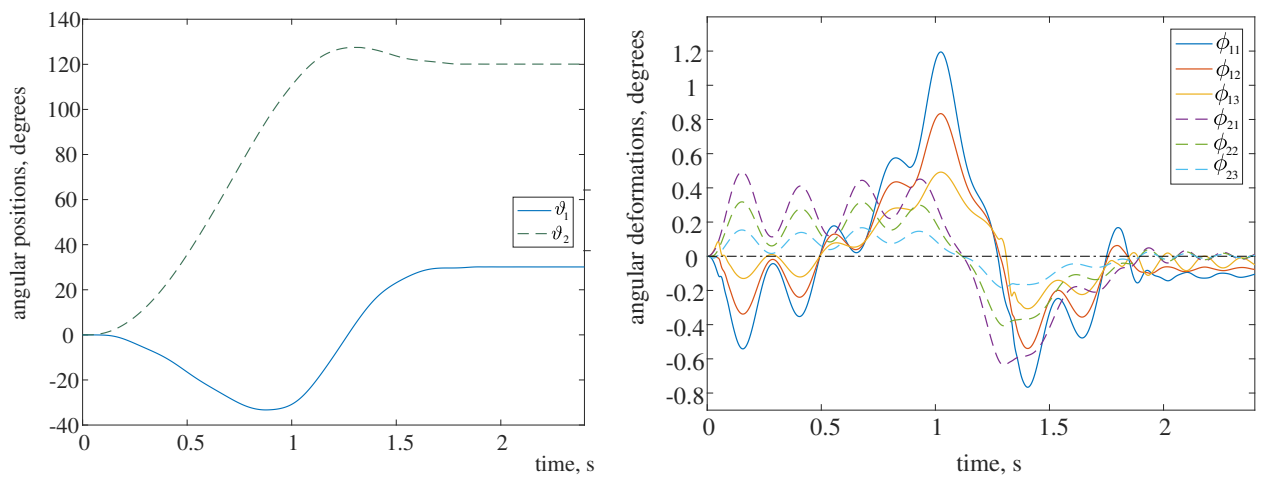

Figure 3. Straight trajectory: angular joint positions (left), and angular link deformations (right).
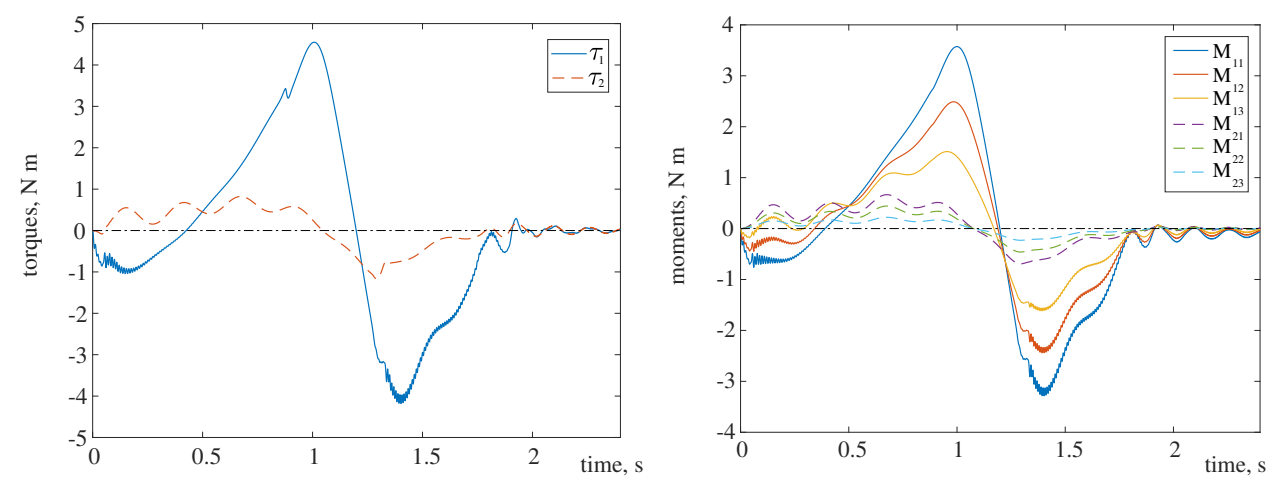

Figure 4. Straight trajectory: joint torques (left), and lumped moments applied to $\varphi_{i j}$ (right).

coefficients are $c_{1}=c_{2}=0.15 \mathrm{Nm} \mathrm{s}$. The parameters related to friction actions are assumed to be the same for the two joints, and specifically they are: the static friction torque $\tau_{S}=0.2 \mathrm{~N} \mathrm{~m}$, the Coulomb friction torque $\tau_{C}=0.1 \mathrm{~N} \mathrm{~m}$, the Stribeck velocity $v_{s}=0.1 \mathrm{rad} / \mathrm{s}$, and the friction coefficients $k_{1}=k_{2}=10^{3} \mathrm{~N} \mathrm{~m}$.

As a first example, we consider the case in which the trajectory of the end effector is rectilinear, as shown by stroboscopic moving pictures in Figure 2, and lasts for $2 \mathrm{~s}$. The control action consists only of torques and moments deriving from (14). The control parameters should be positive for stability reasons, and are set to be $K_{c 1}=K_{c 2}=4 \times 10^{5} \mathrm{~N} / \mathrm{m}$. In Figure 2 the trajectories of the link tips are highlighted in dark red for the actual motion. In particular, in Figure 3, the trajectories of the joint angles $\vartheta_{i}$ and the angular deformations $\varphi_{i j}$ are reported. We note that the angular deformations are very small for the considered trajectory 

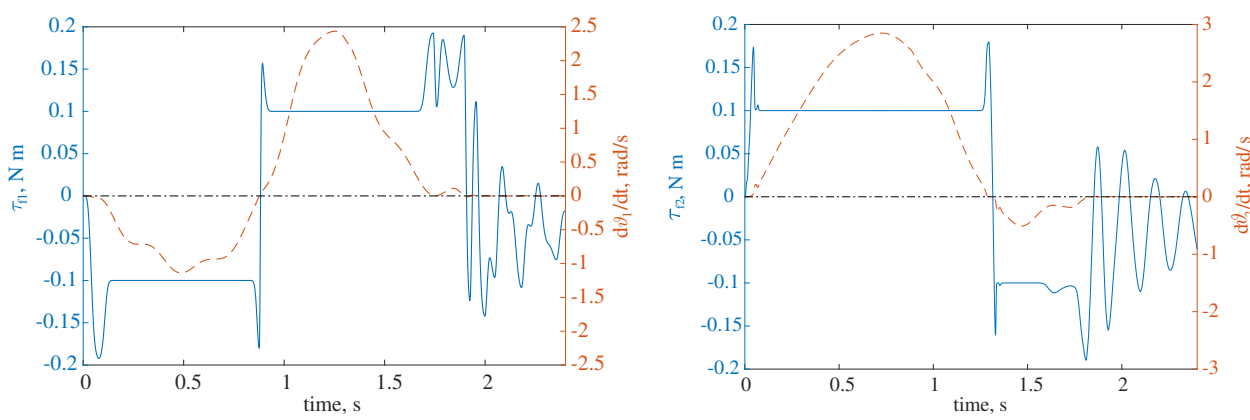

Figure 5. Straight trajectory: friction torque $\tau_{\mathfrak{f} 1}$ and $\dot{\vartheta}_{1}$ (left), and friction torque $\tau_{\mathfrak{f} 2}$ and $\dot{\vartheta}_{2}$ (right).

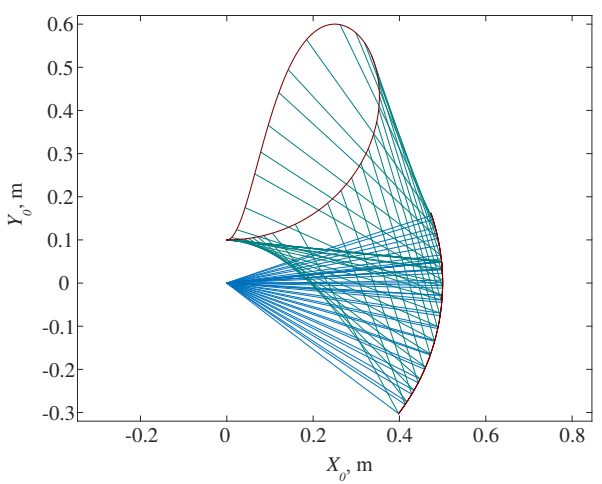

Figure 6. Closed loop trajectory.

planning; indeed, the maximum angular deformation associated to the variable $\varphi_{11}$ is at most 1.2 degrees. The positioning error is not shown since it is negligible.

Figure 4 shows the plots of applied joint torques and the lumped moments on $\varphi_{i j}$, respectively. The nominal trajectory has a settling time of $2 \mathrm{~s}$ in order that from a technological point of view it is possible to consider motors and actuators which are reasonably powerful but not too big. Indeed, the moments required by piezoelectric actuators are easily obtained by exploiting the potentiality provided by the latest technologies.

Figure 5 shows, for the first and second joints, respectively, a comparison between the friction torques and the joint speeds. From this figure it is easy to recognize nonlinear effects such as stick-slip motion and Stribeck effect.

Finally, the case of the closed loop trajectory is considered, as shown in Figure 6. Here, the task time is set to $4 \mathrm{~s}$. Regarding the control action, the control law of the previous case is improved by adding a further damping action; see (16). With this term, possible oscillations of the control actions which can cause some troubles in the real implementation, especially at the beginning, can be avoided. The damping 

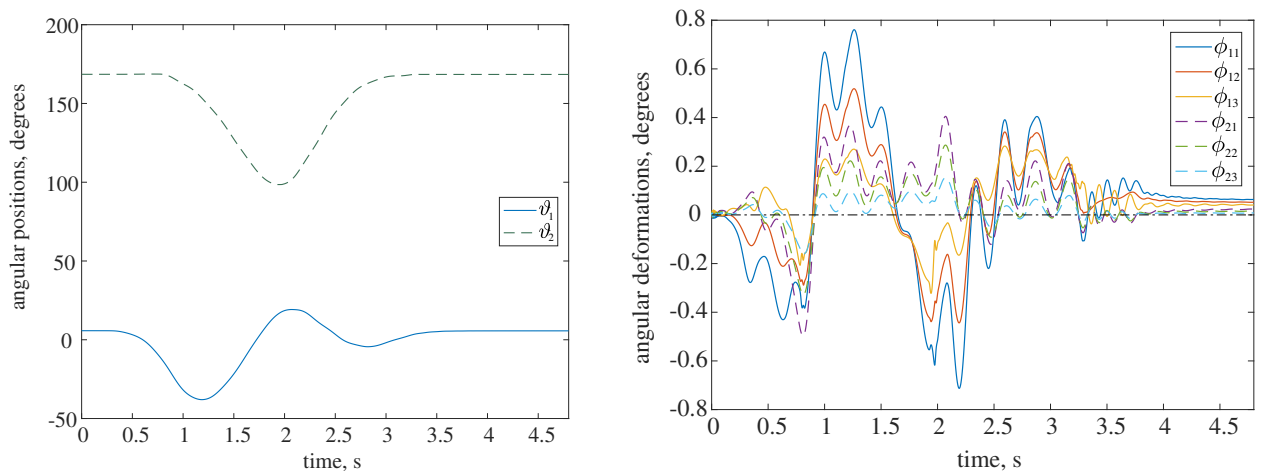

Figure 7. Closed loop trajectory: angular joint positions (left), and angular link deformations (right).
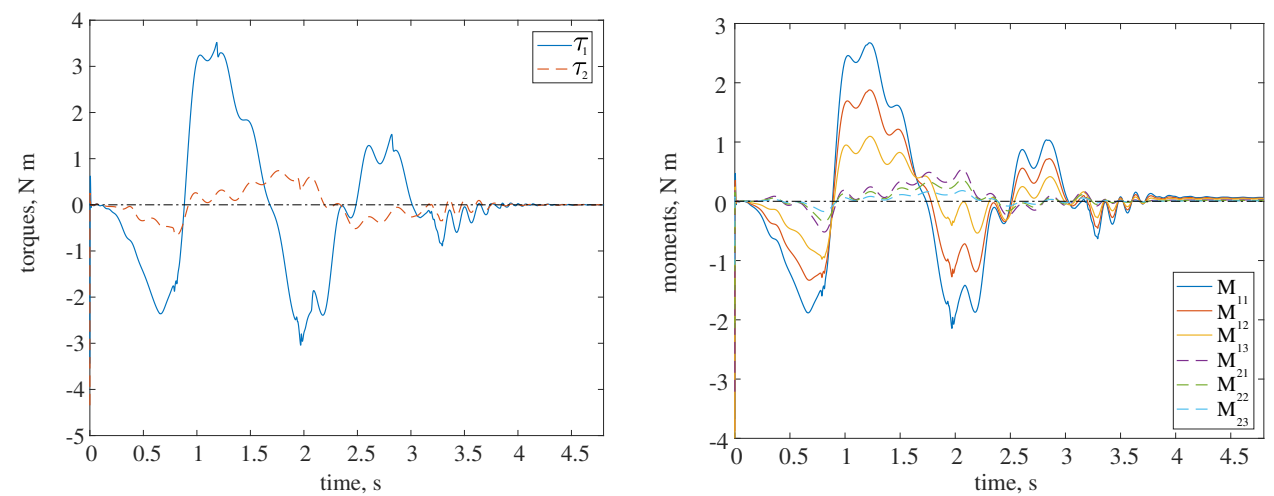

Figure 8. Closed loop trajectory: joint torques (left), and lumped moments (right).

control parameters are set to be $C_{c 1}=C_{c 2}=1 \times 10^{3} \mathrm{~N} \mathrm{~s} / \mathrm{m}$.

Figure 7 shows the joint angles $\vartheta_{i}$ and the angular deformations $\varphi_{i j}$ for the new case. Again, the angular deformations are very small and the maximum angular deformation associated to the variable $\varphi_{11}$ is at most 0.8 degrees. In Figure 8, the applied joint torques and the lumped moments on $\varphi_{i j}$ are plotted.

\section{Conclusions}

In this paper, a planar multilink robot manipulator made up of flexible beams has been modeled by means of a discrete Hencky bar-chain model. An energy-based control has been proposed and validated by numerical simulations.

The use of a Hencky bar-chain approach for space discretization has been motivated in order to consider cases in which the linearization at the basis of the assumed modes method is not feasible. Indeed, the linearization underlying the 
assumed modes method would entail that the motion would be characterized by two distinct time scales: one "slower" for the overall motion and one "faster" for the superimposed vibration. Clearly, this is not possible when dealing with nonlinear systems, as the two time scales are very close and, thus, not neatly distinct.

The finite element method could potentially be suitable for solving such nonlinear problems. Nevertheless, commercial codes which are currently available are still lacking in this respect. Therefore, a homemade code would be needed. The Hencky bar-chain model, for its simplicity and accuracy, has been preferred. Indeed, increasing the number of rigid bars in the discretization results in the Hencky model converging to the nonlinear "elastica" theory. Remarkably, in the case of the proposed control, using a nonlinear model does not entail an excessive computational burden as when employing the computed torque approach which, differently from the energy-based control applied herein to the Hencky bar-chain model, requires the online solution of the whole model.

\section{References}

[Alessandroni et al. 2005] S. Alessandroni, U. Andreaus, F. dell'Isola, and M. Porfiri, "A passive electric controller for multimodal vibrations of thin plates", Comput. Struct. 83:15-16 (2005), $1236-1250$.

[Aminzahed et al. 2017] I. Aminzahed, M. M. Mashhadi, and M. R. V. Sereshk, "Influence of drawn radius in micro deep drawing process of rectangular work pieces via size dependent analysis using piezoelectric actuator", Int. J. Interact. Des. Manuf. 11:4 (2017), 893-902.

[Balobanov et al. 2016] V. Balobanov, S. Khakalo, and J. Niiranen, "Isogeometric analysis of gradientelastic 1D and 2D problems", pp. 37-45 in Generalized continua as models for classical and advanced materials, edited by H. Altenbach and S. Forest, Adv. Struct. Mater. 42, Springer, 2016.

[Baroudi et al. 2019] D. Baroudi, I. Giorgio, A. Battista, E. Turco, and L. A. Igumnov, "Nonlinear dynamics of uniformly loaded Elastica: experimental and numerical evidence of motion around curled stable equilibrium configurations", Z. Angew. Math. Mech. (online publication April 2019).

[Bellezza et al. 1990] F. Bellezza, L. Lanari, and G. Ulivi, "Exact modeling of the flexible slewing link", pp. 734-739 in Proceedings of the 1990 IEEE International Conference on Robotics and Automation (Cincinnati, OH, 1990), vol. 1, IEEE Computer Society, Los Alamitos, CA, 1990.

[Cannon 1984] R. H. Cannon, Jr., "Initial experiments on the end-point control of a flexible one-link robot”, Int. J. Robotics Res. 3:3 (1984), 62-75.

[Cazzani et al. 2016] A. Cazzani, M. Malagù, and E. Turco, "Isogeometric analysis of plane-curved beams", Math. Mech. Solids 21:5 (2016), 562-577.

[Chróścielewski et al. 2019] J. Chróścielewski, R. Schmidt, and V. A. Eremeyev, "Nonlinear finite element modeling of vibration control of plane rod-type structural members with integrated piezoelectric patches", Contin. Mech. Thermodyn. 31:1 (2019), 147-188.

[De Luca and Siciliano 1991] A. De Luca and B. Siciliano, "Closed-form dynamic model of planar multilink lightweight robots”, IEEE Trans. Systems Man Cybernet. 21:4 (1991), 826-839.

[dell'Isola et al. 2016] F. dell'Isola, I. Giorgio, M. Pawlikowski, and N. L. Rizzi, "Large deformations of planar extensible beams and pantographic lattices: heuristic homogenization, experimental and numerical examples of equilibrium”, P. Roy. Soc. London A 472:2185 (2016), 20150790. 
[Dupac and Noroozi 2014] M. Dupac and S. Noroozi, "Dynamic modeling and simulation of a rotating single link flexible robotic manipulator subject to quick stops", Stroj. vestn. J. Mech. Eng. 60:7-8 (2014), 475-482.

[Eugster et al. 2014] S. R. Eugster, C. Hesch, P. Betsch, and C. Glocker, "Director-based beam finite elements relying on the geometrically exact beam theory formulated in skew coordinates", Int. J. Numer. Methods Eng. 97:2 (2014), 111-129.

[Feliu et al. 1992] V. Feliu, K. S. Rattan, and H. B. Brown, "Modeling and control of single-link flexible arms with lumped masses", ASME J. Dyn. Sys. Measure. Cont. 114:1 (1992), 59-69.

[Garcea et al. 1998] G. Garcea, G. A. Trunfio, and R. Casciaro, "Mixed formulation and locking in path-following nonlinear analysis", Comput. Methods Appl. Mech. Eng. 165:1-4 (1998), 247-272.

[Giorgio and Del Vescovo 2018] I. Giorgio and D. Del Vescovo, "Non-linear lumped-parameter modeling of planar multi-link manipulators with highly flexible arms", Robotics 7:4 (2018), 60.

[Giorgio et al. 2019] I. Giorgio, A. Della Corte, and D. Del Vescovo, "Modelling flexible multi-link robots for vibration control: numerical simulations and real-time experiments", Math. Mech. Solids 24:1 (2019), 52-71.

[Greco and Cuomo 2013] L. Greco and M. Cuomo, "B-spline interpolation of Kirchhoff-Love space rods", Comput. Methods Appl. Mech. Eng. 256 (2013), 251-269.

[Greco et al. 2017] L. Greco, M. Cuomo, L. Contrafatto, and S. Gazzo, "An efficient blended mixed B-spline formulation for removing membrane locking in plane curved Kirchhoff rods", Comput. Methods Appl. Mech. Eng. 324 (2017), 476-511.

[Hodges 1990] D. H. Hodges, "A mixed variational formulation based on exact intrinsic equations for dynamics of moving beams", Int. J. Solids Struct. 26:11 (1990), 1253-1273.

[Hughes et al. 2005] T. J. R. Hughes, J. A. Cottrell, and Y. Bazilevs, "Isogeometric analysis: CAD, finite elements, NURBS, exact geometry and mesh refinement", Comput. Methods Appl. Mech. Eng. 194:39-41 (2005), 4135-4195.

[Juang and Phan 1992] J.-N. Juang and M. Phan, "Robust controller designs for second-order dynamic systems: a virtual passive approach", J. Guid. Control Dynam. 15:5 (1992), 1192-1198.

[Khorrami et al. 1991] F. Khorrami, S. Jain, W. Grossman, A. Tzes, and W. Blesser, "Nonlinear control with input preshaping for flexible-link manipulators", pp. 96-101 in Fifth International Conference on Advanced Robotics: Robots in unstructured environments (Pisa, Italy, 1991), vol. 1, IEEE, Piscataway, NJ, 1991.

[Kocsis et al. 2017] A. Kocsis, N. Challamel, and G. Károlyi, "Discrete and nonlocal models of Engesser and Haringx elastica", Int. J. Mech. Sci. 130 (2017), 571-585.

[Lossouarn et al. 2015] B. Lossouarn, J. F. Deü, and M. Aucejo, "Multimodal vibration damping of a beam with a periodic array of piezoelectric patches connected to a passive electrical network", Smart. Mater. Struct. 24:11 (2015), 115037.

[Lumentut and Howard 2015] M. F. Lumentut and I. M. Howard, "Effect of shunted piezoelectric control for tuning piezoelectric power harvesting system responses-analytical techniques", Smart. Mater. Struct. 24:10 (2015), 105029.

[Luongo and D'Annibale 2013] A. Luongo and F. D'Annibale, "Double zero bifurcation of nonlinear viscoelastic beams under conservative and non-conservative loads", Int. J. Non-Linear Mech. 55 (2013), 128-139.

[Pietraszkiewicz and Eremeyev 2009] W. Pietraszkiewicz and V. A. Eremeyev, "On vectorially parameterized natural strain measures of the non-linear Cosserat continuum", Int. J. Solids Struct. 46:11-12 (2009), 2477-2480. 
[Placidi et al. 2017] L. Placidi, E. Barchiesi, and A. Battista, "An inverse method to get further analytical solutions for a class of metamaterials aimed to validate numerical integrations", pp. 193210 in Mathematical modelling in solid mechanics, edited by F. dell'Isola et al., Adv. Struct. Mater. 69, Springer, 2017.

[Ramachandran et al. 1992] S. Ramachandran, T. Nagarajan, and N. Siva Prasad, "A finite element approach to the design and dynamic analysis of platform type robot manipulators", Finite Elem. Anal. Des. 10:4 (1992), 335-350.

[Rosi et al. 2018] G. Rosi, L. Placidi, and N. Auffray, "On the validity range of strain-gradient elasticity: a mixed static-dynamic identification procedure”, Eur. J. Mech. A Solids 69 (2018), 179-191.

[Rubinstein 1999] D. Rubinstein, "Dynamics of a flexible beam and a system of rigid rods, with fully inverse (one-sided) boundary conditions", Comput. Methods Appl. Mech. Eng. 175:1-2 (1999), 87-97.

[Sharf 1996] I. Sharf, "Geometrically non-linear beam element for dynamics simulation of multibody systems", Int. J. Numer. Methods Eng.. 39:5 (1996), 763-786.

[Spagnuolo and Andreaus 2019] M. Spagnuolo and U. Andreaus, "A targeted review on large deformations of planar elastic beams: extensibility, distributed loads, buckling and post-buckling", Math. Mech. Solids 24:1 (2019), 258-280.

[Theodore and Ghosal 1995] R. J. Theodore and A. Ghosal, "Comparison of the assumed modes and finite element models for flexible multilink manipulators", Int. J. Rob. Res. 14:2 (1995), 91-111.

[Turco 2018] E. Turco, "Discrete is it enough? The revival of Piola-Hencky keynotes to analyze three-dimensional elastica", Contin. Mech. Thermodyn. 30:5 (2018), 1039-1057.

[Turco et al. 2016] E. Turco, F. dell'Isola, A. Cazzani, and N. L. Rizzi, "Hencky-type discrete model for pantographic structures: numerical comparison with second gradient continuum models", $Z$. Angew. Math. Phys. 67:4 (2016), 85.

[Wang et al. 2015] C. M. Wang, H. Zhang, R. P. Gao, W. H. Duan, and N. Challamel, "Hencky bar-chain model for buckling and vibration of beams with elastic end restraints", Int. J. Struct. Stab. Dyn. 15:7 (2015), 1540007.

[Weeger et al. 2013] O. Weeger, U. Wever, and B. Simeon, "Isogeometric analysis of nonlinear Euler-Bernoulli beam vibrations", Nonlinear Dynam. 72:4 (2013), 813-835.

[Canudas de Wit et al. 1995] C. Canudas de Wit, H. Olsson, K. J. Åström, and P. Lischinsky, "A new model for control of systems with friction", IEEE Trans. Automat. Control 40:3 (1995), 419-425.

[Yildizdag et al. 2018] M. E. Yildizdag, M. Demirtas, and A. Ergin, "Multipatch discontinuous Galerkin isogeometric analysis of composite laminates", Contin. Mech. Thermodyn. (online publication July 2018).

Received 27 Dec 2018. Revised 17 Feb 2019. Accepted 20 Mar 2019.

IVAN GIORGIO: ivan.giorgio@uniroma1.it

Department of Mechanical and Aerospace Engineering, Università di Roma "La Sapienza", Italy and

International Research Center on Mathematics and Mechanics of Complex Systems, Università degli studi dell'Aquila, Italy

Dionisio Del Vescovo: dionisio.delvescovo@uniroma1.it

Department of Mechanical and Aerospace Engineering, Università di Roma "La Sapienza", Italy and

International Research Center on Mathematics and Mechanics of Complex Systems, Università degli studi dell'A quila, Italy 
EDITORIAL BOARD

ANTONIO CARCATERRA

ERIC A. CARLEN

FRANCESCO DELL'ISOLA

RAFFAELE ESPOSITO

ALBERT FANNJIANG

Gilles A. FrancFort

Pierangelo Marcati

JEAN-JACQUES MARIGO

PETER A. MARKOWICH

MARTIN OSTOJA-STARZEWSKI

PIERRE SEPPECHER

DAVID J. STEIGMANN

PAUL STEINMANN

PierRe M. SuQueT

MANAGING EDITORS

MICOL AMAR

ANGELA MADEO

MARTIN OSTOJA-STARZEWSKI

\section{ADVISORY BOARD}

ADNAN AKAY

Holm AltenbaCH

MICOL AMAR

HARM ASKES

TEODOR ATANACKOVIĆ

VICTOR BERDICHEVSKY

GuY BouchitTÉ

ANDREA BRAIDES

ROBERTO CAMASSA

MAURO CARFORE

ERIC DARVE

FELIX DARVE

ANNA DE MASI

Gianpietro Del Piero

Emmanuele Di Benedetto

VICTOR A. EREMEYEV

BERNOLD FIEDLER

IRENE M. GAMBA

DAVID Y. GAO

SERGEy GaVRILYUK

Timothy J. HeAley

DOMINIQUE JEULIN

ROgER E. KHAYAT

CORRADO LATTANZIO

ROBERT P. LIPTON

ANGELO LUONGO

ANGEla MADEO

JUAN J. MANFREDI

CARlo MARCHIORO

ANIL MISRA

ROBERTO NATALINI

PATRIZIO NEFF

Thomas J. Pence

ANDREY PIATNITSKI

ERRICO PRESUTtI

MARIO PUlVIRENTI

Lucio Russo

Miguel A. F. SANJUAN

PATRICK SElvaduraI

MIROSLAV ŠILHAVÝ

GUIDO SWEERS

ANTOINETTE TORDESILLAS

LEV TRUSKINOVSKY

JUAN J. L. VELÁZQUEZ

VINCENZO VESPRI

ANGELO VULPIANI msp.org/memocs

Università di Roma "La Sapienza", Italia

Rutgers University, USA

(CO-CHAIR) Università di Roma "La Sapienza", Italia

(TREASURER) Università dell'Aquila, Italia

University of California at Davis, USA

(CO-CHAIR) Université Paris-Nord, France

Università dell' Aquila, Italy

École Polytechnique, France

DAMTP Cambridge, UK, and University of Vienna, Austria

(CHAIR MANAGING EDITOR) Univ. of Illinois at Urbana-Champaign, USA

Université du Sud Toulon-Var, France

University of California at Berkeley, USA

Universität Erlangen-Nürnberg, Germany

LMA CNRS Marseille, France

Università di Roma "La Sapienza", Italia

Université de Lyon-INSA (Institut National des Sciences Appliquées), France (CHAIR MANAGING EDITOR) Univ. of Illinois at Urbana-Champaign, USA

Carnegie Mellon University, USA, and Bilkent University, Turkey

Otto-von-Guericke-Universität Magdeburg, Germany

Università di Roma "La Sapienza”, Italia

University of Sheffield, UK

University of Novi Sad, Serbia

Wayne State University, USA

Université du Sud Toulon-Var, France

Università di Roma Tor Vergata, Italia

University of North Carolina at Chapel Hill, USA

Università di Pavia, Italia

Stanford University, USA

Institut Polytechnique de Grenoble, France

Università dell' Aquila, Italia

Università di Ferrara and International Research Center MEMOCS, Italia

Vanderbilt University, USA

Gdansk University of Technology, Poland

Freie Universität Berlin, Germany

University of Texas at Austin, USA

Federation University and Australian National University, Australia

Université Aix-Marseille, France

Cornell University, USA

École des Mines, France

University of Western Ontario, Canada

Università dell' Aquila, Italy

Louisiana State University, USA

Università dell'Aquila, Italia

Université de Lyon-INSA (Institut National des Sciences Appliquées), France University of Pittsburgh, USA

Università di Roma "La Sapienza", Italia

University of Kansas, USA

Istituto per le Applicazioni del Calcolo "M. Picone", Italy

Universität Duisburg-Essen, Germany

Michigan State University, USA

Narvik University College, Norway, Russia

Università di Roma Tor Vergata, Italy

Università di Roma “La Sapienza”, Italia

Università di Roma “Tor Vergata", Italia

Universidad Rey Juan Carlos, Madrid, Spain

McGill University, Canada

Academy of Sciences of the Czech Republic

Universität zu Köln, Germany

University of Melbourne, Australia

École Polytechnique, France

Bonn University, Germany

Università di Firenze, Italia

Università di Roma La Sapienza, Italia

MEMOCS (ISSN 2325-3444 electronic, 2326-7186 printed) is a journal of the International Research Center for the Mathematics and Mechanics of Complex Systems at the Università dell'Aquila, Italy.

Cover image: "Tangle” by $\odot$ John Horigan; produced using the Context Free program (contextfreeart.org).

PUBLISHED BY

7 mathematical sciences publishers

nonprofit scientific publishing

http://msp.org/

(C) 2019 Mathematical Sciences Publishers 
Mathematics and Mechanics of Complex Systems vol. 7 no. 2

A polynomial chaos expanded hybrid fuzzy-stochastic model for transversely fiber reinforced plastics

Eduard Penner, Ismail Caylak, Alex Dridger and Rolf Mahnken

Dynamic boundary conditions for membranes whose surface energy depends on the mean and Gaussian curvatures

Sergey Gavrilyuk and Henri Gouin Energy-based trajectory tracking and vibration control for multilink highly flexible manipulators

Ivan Giorgio and Dionisio Del Vescovo

A model of the proppant flowback: setup of the theoretical framework

Ksenia P. Frolova, Polina M. Grigoreva, Konstantin E.

Lezhnev and Grigoriy V. Paderin

The object detection by autonomous apparatus as a solution of the 189 Buffon needle problem

Mikhail A. Guzev, Gurami S. Tsitsiashvili and Marina A.

Osipova

MEMOCS is a journal of the International Research Center for the Mathematics and Mechanics of Complex Systems at the Università dell' Aquila, Italy.

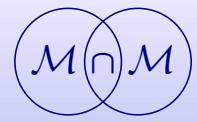

